\title{
Video Article \\ An Orbital Shaking Culture of Mammalian Cells in O-shaped Vessels to Produce Uniform Aggregates
}

\author{
Ikki Horiguchi $^{1,2}$, Ikumi Suzuki ${ }^{3}$, Takashi Morimura ${ }^{3}$, Yasuyuki Sakai ${ }^{1,4}$ \\ ${ }^{1}$ Department of Chemical System Engineering, University of Tokyo \\ ${ }^{2}$ Department of Biotechnology, Osaka University \\ ${ }^{3}$ Biotech Business Unit, Fukoku Co. Ltd \\ ${ }^{4}$ Institute of Industrial Science, University of Tokyo
}

Correspondence to: Ikki Horiguchi at horiguchi@bio.eng.osaka-u.ac.jp, Ikumi Suzuki at i_suzuki@fukoku-rubber.co.jp

URL: https://www.jove.com/video/57922

DOI: doi:10.3791/57922

Keywords: Bioengineering, Issue 143, Suspension culture, orbital shaking, mass production, mammalian cell, culture bag, aggregate

Date Published: 1/7/2019

Citation: Horiguchi, I., Suzuki, I., Morimura, T., Sakai, Y. An Orbital Shaking Culture of Mammalian Cells in O-shaped Vessels to Produce Uniform Aggregates. J. Vis. Exp. (143), e57922, doi:10.3791/57922 (2019).

\section{Abstract}

Suspension cultures of mammalian cell aggregates are required for various applications in medical and biotechnological fields. The disposable bag-based method is one of the simplest techniques for the mass production of cellular aggregates, but it does not protect the cultures against over-aggregation, which occurs when they gather at the bottom center of the culture vessel. To solve this problem, we developed an O-shaped dish and an O-shaped bag, neither of which contains a central region. Aggregates grown in either O-shaped culture vessel were noticeably more uniform in size than aggregates grown in conventional vessels. Histological analyses showed that aggregates in conventional culture dishes contained necrotic cores most likely caused by a poor oxygen supply. In contrast, aggregates that were grown in the O-shaped bag, even those with similar diameters to aggregates in conventional culture dishes, did not show necrotic cores. These results suggest that the O-shaped bag provides sufficient oxygen to the aggregates due to the oxygen permeability of the bag material. We, therefore, propose that this novel gaspermeable $\mathrm{O}$-shaped culture bag is suitable for the mass production of uniform aggregates that are necessary in various biotechnological fields.

\section{Video Link}

The video component of this article can be found at https://www.jove.com/video/57922/

\section{Introduction}

Suspension cell culture plays an important role in the cell production for regenerative medicine ${ }^{1}$ and recombinant protein production ${ }^{2}$ because it is easy to scale up and achieve high cell densities, sometimes up to more than $10^{7}$ cells $/ \mathrm{mL}^{5}$. Chinese hamster ovary (CHO) cells ${ }^{3}$ and human embryonic kidney cells 293 (HEK293) have been grown in suspension culture, and human pluripotent stem cells (hPSCs) have recently been grown in suspension culture systems for regenerative therapies ${ }^{1,6,7}$. The use of suspension cultures is expected to increase in the future.

In suspension culture, some cell lines cannot grow as single cells and must, therefore, form aggregates. For example, hPSCs cannot survive in suspension conditions without forming aggregates. However, this requirement for aggregated growth presents difficulties for uniform suspension cultures. One difficulty is the formation of uniform aggregates in the early period of the culture, which determines the efficiency of the suspension culture ${ }^{8}$. Another difficulty is the mass transfer of nutrients into aggregates. In particular, the oxygen supply limits the maximum size of aggregates, and a poor oxygen supply causes necrosis in the center of aggregates ${ }^{9}$. Consequently, suspension cultures of cell aggregates are more difficult to obtain than conventional suspension cultures. Nevertheless, suspension cultures are crucial for biomedical and biotechnology applications.

Orbital shaking vessels are one of the simplest suspension culture systems that achieve culture medium mixtures without impeller agitation. Shear stress from the impeller and the dynamic medium flow is the major problem of suspension culture because it causes cell damage and differentiation. To achieve agitation with a lower shear stress, researchers and industries have developed a variety of orbital shaking vessel systems $^{2,10}$.

However, conventional culture vessels are not designed for orbital shaking cultures. In orbital shaking vessels, cells gravitate towards the centerbottom of the vessels by a type of medium flow known as the "Einstein's tea leaf paradox" ${ }^{11}$, which causes the inhomogeneous aggregation of cells. The circular flow, caused by a centrifugal force and a friction between the culture medium and a vessel, sweeps cells into the center ${ }^{11,12}$. In addition, conventional culture bags for mass culture are square-shaped, which is not suitable for orbital shaking.

In this study, we developed a novel culture bag suitable for orbital shaking cultures. The novelty of this bag is its O-shape which does not have a center region, so cells are prevented from gathering at the bottom center region. We have also demonstrated the handling of these vessels with an HEK293 culture to demonstrate the possibility of these bags for biotechnological applications. 


\section{Protocol}

\section{Preparation of the Cells and Materials}

1. Cultivate HEK293 cells in Dulbecco's modified Eagle medium with a $10 \%$ fetal bovine serum (FBS) and $1 \%$ non-essential amino acids. Adjust the $\mathrm{pH}$ of the culture medium to 6.8 - 7.6.

2. After culturing for 5 to $7 \mathrm{~d}$, dissociate the cells with a $0.25 \%$ trypsin-ethylenediaminetetraacetic acid solution (trypsin-EDTA) and reseed the cells at $5,000-10,000$ cells $/ \mathrm{cm}^{2}$.

\section{Seeding the Cells in an O-shaped Bag}

1. Dissociate the cells as described in step 1.2 and resuspend them in $2-5 \mathrm{~mL}$ of culture medium.

2. Filter the cell suspension through a cell strainer $(40 \mu \mathrm{m})$ to collect a single cell suspension.

3. Count the number of cells by trypan blue staining and automatic cell counter, and then prepare $20 \mathrm{~mL}$ of the cell suspension $\left(2.0 \times 10^{5}\right.$ cells/ $\mathrm{mL})$.

4. Connect the inlet of the O-shaped bag (Figure 1a) to a clamped $50 \mathrm{~mL}$ syringe without a plunger.
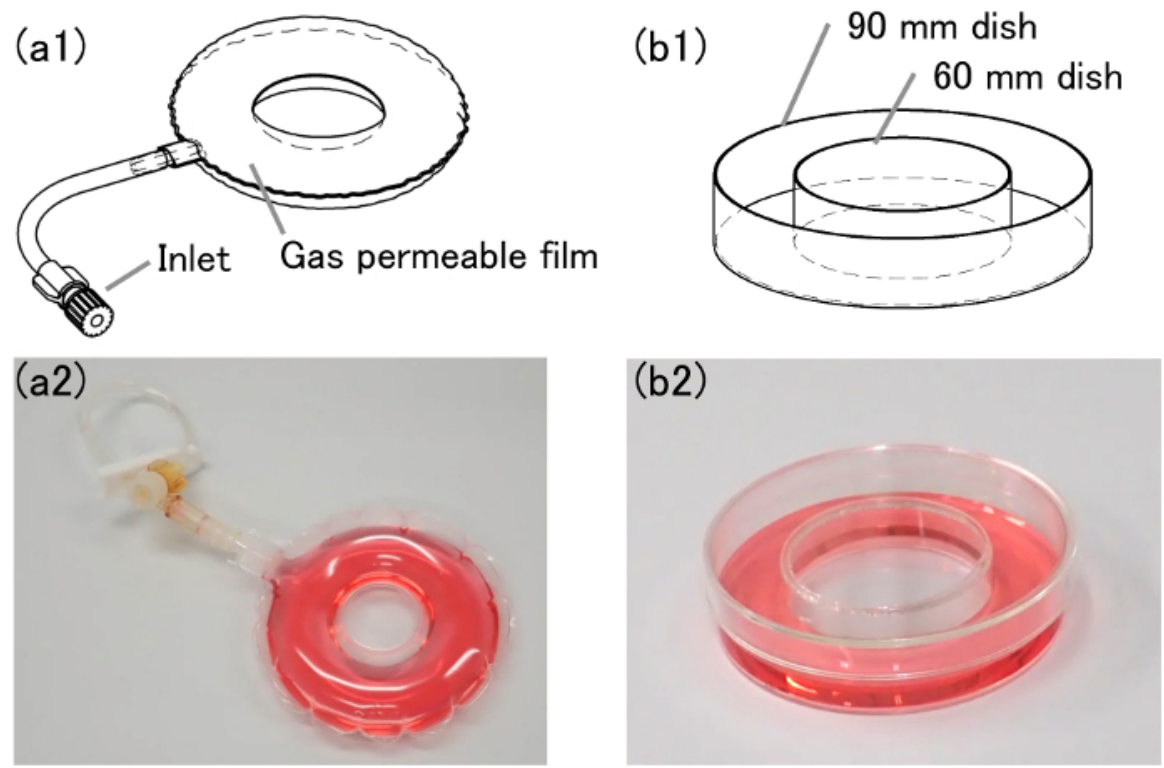

Figure 1: Schematic images and pictures of O-shaped vessels. (a1) This is a schematic image of an O-shaped bag. (a2) This is a picture of an O-shaped bag. (b1) This is a schematic image of an O-shaped dish. (b2) This is a picture of an O-shaped dish. The outer and inner diameter of the O-shaped bag are $90 \mathrm{~mm}$ and $20 \mathrm{~mm}$, respectively. Please click here to view a larger version of this figure.

5. Pipette the prepared cell suspension into the O-shaped bag through the clamped syringe.

6. Replace the first clamped syringe with a clean syringe. Add $55 \mathrm{~mL}$ of clean air through the clean syringe to expand the bag completely.

7. Clamp the inlet tube and then close the inlet. Finally, remove the clamp from the tube.

8. Incubate the cells in the O-shaped bag by shaking them at $45 \mathrm{rpm}$ in conditions of $37^{\circ} \mathrm{C}$ and $5 \% \mathrm{CO}_{2}$.

\section{Medium Change (optional)}

1. Transfer the cell suspension into a $50 \mathrm{~mL}$ tube through the inlet.

2. Centrifuge it for $2 \mathrm{~min}$ at $200 \mathrm{xg}$ at room temperature, and then aspirate the supernatant.

3. Add $20 \mathrm{~mL}$ of culture medium and resuspend the cells.

4. Add the resuspended cells to the O-shaped bag by following steps $2.4-2.7$.

5. Incubate the cells in the O-shaped bag by shaking them at $45 \mathrm{rpm}$ in conditions of $37^{\circ} \mathrm{C}$ and $5 \% \mathrm{CO}_{2}$.

\section{Collecting the Cells from the Bag}

1. Transfer the cell suspension into a $50 \mathrm{~mL}$ tube through the inlet.

2. Wash the inside of the bag with $20 \mathrm{~mL}$ of calcium/magnesium-free phosphate buffered saline [PBS(-), $\mathrm{pH}=7.4-7.6]$ and then drain the contents into a tube to collect the remaining cells from the bag.

3. Centrifuge the collected cell suspension for $3 \mathrm{~min}$ at $200 \mathrm{xg}$ at room temperature, and then aspirate the supernatant.

4. Add $10 \mathrm{~mL}$ of PBS(-) and wash the aggregates.

5. Centrifuge them for $3 \mathrm{~min}$ at $200 \mathrm{xg}$ at room temperature, and then aspirate the supernatant to collect the aggregates. 
6. Add $4 \mathrm{~mL}$ of PBS and $1 \mathrm{~mL}$ of trypsin and incubate them with the aggregates for $10 \mathrm{~min}$ at $37^{\circ} \mathrm{C}$ to dissociate the cells for counting (optional).

\section{Preparation of an O-shaped Dish (optional)}

NOTE: See Figure $1 \mathbf{b}$ for a schematic image of the O-shaped dish.

1. Cut out the bottom of a $60 \mathrm{~mm}$ or $35 \mathrm{~mm}$ dish with a hot knife and utilize it as an inner dish.

2. Put the $60 \mathrm{~mm}$ dish upside-down on the center of a $100 \mathrm{~mm}$ dish. NOTE: A guide sheet can help to decide the position of the $60 \mathrm{~mm}$ dish.

3. If required, put viscosity-adjusted cyclohexanone on the commissure from the inside of $60 \mathrm{~mm}$ dish.

4. Dry the O-shaped dish for a few days and sterilize it by gamma ray or ethylene oxide gas.

\section{Representative Results}

According to our measurements, aggregates grown in the conventional dish had varied diameters after $5 \mathrm{~d}$ of orbital shaking. In contrast, aggregates grown in O-shaped vessels for $5 \mathrm{~d}$ had much more uniform diameters. The conventional dish culture showed small aggregates $(50-200 \mu \mathrm{m})$, whereas the cultures in the O-shaped vessels did not contain any small aggregates (Figure 2a). According to the image-based size measurement, the conventional dish culture showed two different peaks (Figure 2b1), indicating a wide deviation of aggregate size. This result implied that aggregates in the conventional dish were growing under two different conditions in the same culture, which could result in heterogeneous cell quality. On the other hand, aggregates in O-shaped vessels showed a single peak and less deviation in diameter than those in the conventional dish (Figure $\mathbf{2 b 2}$ and $\mathbf{2 b 3}$ ), suggesting that such aggregates may be of more uniform quality.
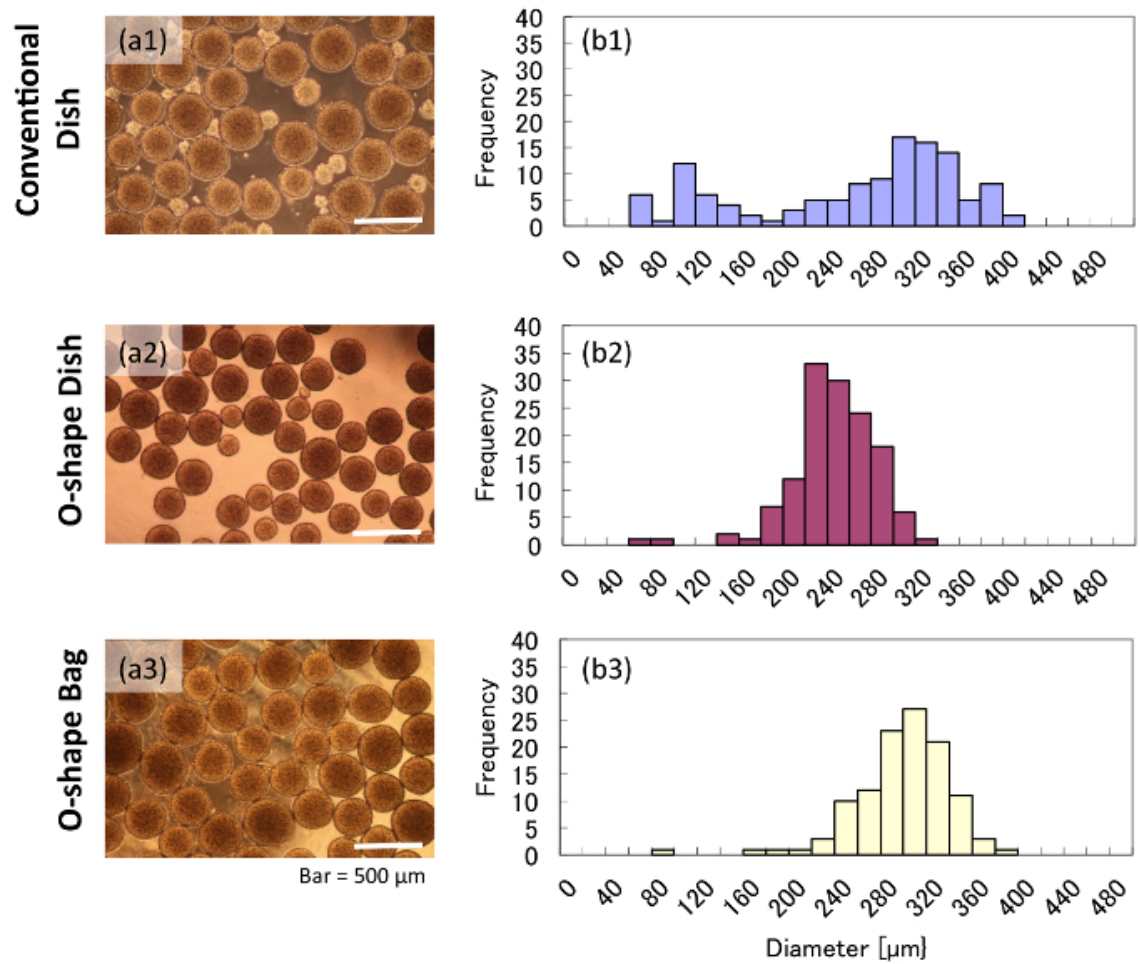

Figure 2: Morphologies and diameters of aggregates grown in various vessels. These panels show (a) brightfield images and (b) histograms of aggregates grown in either (a1 and b1) a conventional dish, (a2 and b2) an O-shaped dish, or (a3 and b3) an O-shaped bag. Please click here to view a larger version of this figure.

Hematoxylin and eosin staining of aggregate cross-sections showed that aggregates grown in the conventional dish had some denucleated cells and necrotic cores (Figure 3a). However, aggregates grown in O-shaped vessels did not show any necrotic cores (Figure 3b and 3c). In particular, aggregates grown in the O-shaped bag were as large as those in the conventional dish yet did not have any necrotic cores (Figure 3c). These results suggested that the gas-permeable bag supplied enough oxygen to the culture. 
(a) Conventional Dish

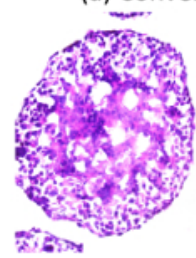

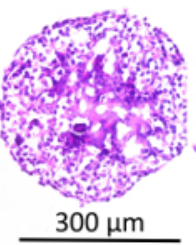

(b) O-shape Dish
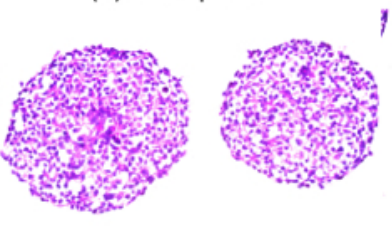

(c) O-shape Bag

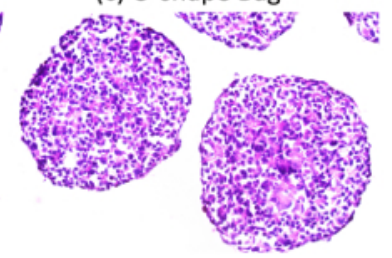

Figure 3: Cross-sections of aggregates in various vessels. The cross-sections are $12 \mu \mathrm{m}$ thick and were prepared from frozen samples. The sections were stained with hematoxylin and eosin to visualize the cells. (a) The cell aggregates grown in a conventional suspension culture showed necrotic cores. Cell aggregates grown in either (b) an O-shaped dish or (c) an O-shaped bag showed very little necrosis in their cores. Please click here to view a larger version of this figure.

Cell counts showed that more than $85 \%$ of the cells survived for $5 \mathrm{~d}$ of suspension culture in each vessel (Figure $4 \mathrm{a}$ ). The final cell density was approximately $1.5-2.0 \times 10^{6} \mathrm{cells} / \mathrm{mL}$. Although there was no significant difference, the growth ratio in the O-shaped bag was higher than those in other vessels (Figure 4b). The specific growth rates of the cells in the conventional dish, the O-shaped dish, and the O-shaped bag between day 2 and day 5 were $0.018,0.025$, and $0.020 \mathrm{~h}^{-1}$, respectively.

(a) Cell Viability

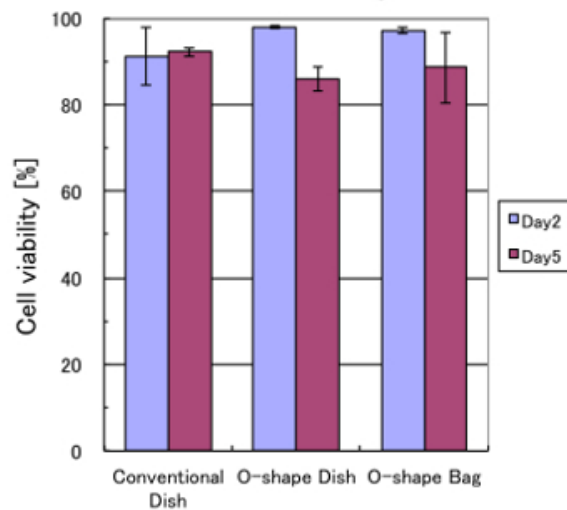

(b) Fold Increase

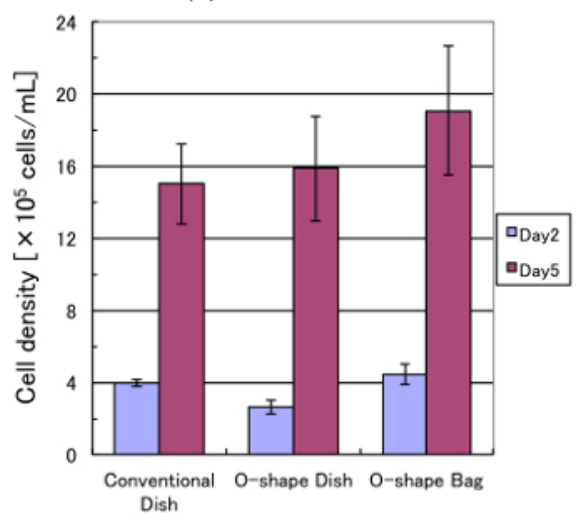

Figure 4: Cell viability and growth. These panels show the (a) cell viability and (b) cell density of HEK293 cells in various culture vessels after $2 \mathrm{~d}$ of culture (day 2) and $5 \mathrm{~d}$ of culture (day 5). The values shown represent the mean of the results from 3 independent experiments. The values for each experiment were calculated from trypan blue-stained cells counted with an automatic cell counter. The error bars indicate standard deviation. Please click here to view a larger version of this figure.

Supplemental Figure 1: Bead distribution in 2 different dish formats in various shaking conditions. This panel shows the bead distribution during various shaking conditions $(30,40$, and $45 \mathrm{rpm})$ in a conventional and O-shaped dish. Please click here to download this file.

\section{Discussion}

In this study, we developed O-shaped vessels and performed an HEK293 suspension culture in them for a uniform aggregates formation and expansion. In the conventional culture dish, an orbital shaking culture produced two different diameters of aggregates, whereas we observed uniform aggregates in the O-shaped vessels (Figure 1). According to the observation of the distribution of stained beads in the orbital shaking conditions, beads gather in the center-bottom of a conventional culture dish. That gathering presumably caused the difference in cell density leading to various sizes of aggregates. Alternatively, beads were distributed in the O-shaped dish which does not have the center-bottom region (Supplemental Figure 1). This distribution probably causes the uniformly-sized aggregates in O-shaped vessels. Another-widely usedapproach to producing uniform aggregates is the culturing in microwells, but this approach has some problems, such as in supplying a culture medium without aggregates dropping out of the microwells ${ }^{13}$. In the case of O-shaped vessels, uniform aggregates can be produced in a simple suspension culture.

The orbital shaking culture is a popular culture system utilized for mammalian cells. There are various culture methods for the mass production of mammalian cells, such as the stirred tank bioreactor ${ }^{14}$, wave-motioned bags ${ }^{15}$, and rotating bottles. Orbital shaking cultures do not include an inner impeller for stirring the medium, unlike the stirred tank bioreactor does. This feature is similar to the wave-motioned bags and rotating bottles. These impeller-free culture systems can avoid cellular damage from the shear force surrounding the impellers and realize low shear stress in suspension culture. Especially, orbital shaking culture systems are effective for the mass production of sensitive cells such as mammalian cells because of their high scalability and low shear stress.

O-shaped vessels can improve the remaining problem of orbital shaking culture in the formation of aggregates. In orbital shaking culture systems, floating cells migrate to the center and bottom of the vessel, which is known as the "Einstein's tea leaf paradox"11. This migration causes the inhomogeneous aggregation and non-uniform aggregate production in conventional orbital shaking vessels. In this study, O-shaped vessels prevented the concentration of cells into the center-bottom of the vessels, which is speculated as the reason of uniform aggregation in orbital shaking O-shaped vessels. 
Histological analyses showed that the aggregates in the conventional dish contained denucleated cells (Figure 2a). In contrast, denucleated cells did not appear in the aggregates from O-shaped vessels (Figure $\mathbf{2 b}$ and $\mathbf{2 c}$ ). It is possible that these denucleated cells were caused by a shortage of substrates such as glucose, glutamine, and oxygen ${ }^{9}$. According to the size measurement, aggregates in the O-shaped vessels had homogeneous diameters lower than $400 \mu \mathrm{m}$. In contrast, in a conventional dish, some aggregates had a diameter larger than $400 \mu \mathrm{m}$, and these aggregates included denucleated cells. This result suggests that creating homogeneous-sized aggregates in O-shaped vessels is effective in controlling the quality of aggregates. In addition, it is also speculated that the oxygenation through the gas-permeable polyethylene film prevented the appearance of denucleated cells in the O-shaped bag.

These experiments showed the possibility of these O-shaped vessels as a simple system for producing uniform aggregates. Although other culture bags for suspension culture have been developed ${ }^{15}$, those culture bags are square-shaped, which prevents the culture from getting effectively mixed in orbital shaking. The bag in this study has a novel round shape suitable for orbital shaking to produce aggregates with a homogeneous size. This characteristic of vessels is important for controlling the conditions and the high reproducibility of cells in mass production. The possible application of the O-shaped vessel is widespread. It can be used when producing recombinant proteins from cells and for regenerative medicine when dealing with stem cells.

In conclusion, we developed a novel O-shaped bag suitable for producing uniform cell aggregates with an orbital shaking culture. The bag shows possibilities for various biomedical applications such as in regenerative medicine.

\section{Disclosures}

The authors have nothing to disclose.

\section{Acknowledgements}

This research is supported by a collaboration with FUKOKU, CO., Ltd. Takao Yoshida from FUKOKU, CO., Ltd. provided the idea of the Oshaped culture bag. Takamasa Sato from FUKOKU, CO., Ltd. supported this research in terms of a computational simulation for developing the O-shaped culture bag. We would like to appreciate the corresponding author's current affiliation, Osaka University, for allowing us to work on the publication.

\section{References}

1. Mihara, Y. et al. Production of pancreatic progenitor cells from human induced pluripotent stem cells using a three-dimensional suspension bioreactor system. Journal of Tissue Engineering and Regenerative Medicine. 11 (11): 3193-3201 (2017).

2. Raven, N. et al. Scaled-up manufacturing of recombinant antibodies produced by plant cells in a $200-\mathrm{L}$ orbitally-shaken disposable bioreactor. Biotechnology and Bioengineering. 112 (2), 308-321 (2015).

3. Han, Y. et al. Cultivation of recombinant chinese hamster ovary cells grown as suspended aggregates in stirred vessels. Journal of Bioscience and Bioengineering. 102 (5), 430-435 (2006).

4. Portolano, N. et al. Recombinant Protein Expression for Structural Biology in HEK 293F Suspension Cells: A Novel and Accessible Approach. Journal of Visualized Experiments. (92), e51897 (2014).

5. Himmelfarb, P., Thayer, P.S., Martin, H.E. Spin filter culture: the propagation of mammalian cells in suspension. Science. 164 (3879), $555-557$ (1969).

6. Kropp, C., Massai, D., Zweigerdt, R. Progress and challenges in large-scale expansion of human pluripotent stem cells. Process Biochemistry. 59, 244-254 (2017).

7. Singh, H., Mok, P., Balakrishnan, T., Rahmat, S. N. B., Zweigerdt, R. Up-scaling single cell-inoculated suspension culture of human embryonic stem cells. Stem Cell Research. 4 (3), 165-179 (2010).

8. Horiguchi, I., Sakai, Y. Serum replacement with albumin-associated lipids prevents excess aggregation and enhances growth of induced pluripotent stem cells in suspension culture. Biotechnology Progress. 32 (4), 1009-1016 (2016).

9. Sutherland, R.M. et al. Oxygenation and differentiation in multicellular spheroids of human colon carcinoma. Cancer Research. 46 (10), 5320-5329 (1986).

10. Hang, H. et al. Computational fluid dynamics modeling of an inverted frustoconical shaking bioreactor for mammalian cell suspension culture. Biotechnology and Bioprocess Engineering. 16 (3), 567-575 (2011).

11. Einstein, A. The cause of the formation of meanders in the courses of rivers annd of the so-called bear's law. Die Naturwissenschaften. 14 (1926).

12. Tandon, A., Dartmouth, U., Marshall, J. Einstein's tea leaves and pressure systems in the atmosphere. The Physics Teacher. 48, 292-295 (2010).

13. Miyamoto, D., Nakazawa, K. Differentiation of mouse iPS cells is dependent on embryoid body size in microwell chip culture. Journal of Bioscience and Bioengineering. 122 (4), 507-512 (2016).

14. Olmer, R. et al. Suspension culture of human pluripotent stem cells in cotrolled, stirred bioreactors. Tissue Engineering Part C: Methods. 18 (10), 772-784 (2012).

15. Singh, V. Disposable bioreactor for cell culture using wave-induced agitation. Cytotechnology. 30, 149-158 (1999). 